\title{
Turbulence characterization and image processing data sets from a NATO RTO SET 165 trial in Dayton $(\mathrm{OH})$
}

\author{
M.-T. Velluet ${ }^{\mathrm{a}}$, Mikhail Vorontsov ${ }^{\mathrm{b}}$, Piet Schwering ${ }^{\mathrm{c}}$, Gabriele Marchi $^{\mathrm{d}}$, Stephane Nicolas ${ }^{\mathrm{e}}$, \\ Jim Riker $^{\mathrm{f}}$ \\ ${ }^{a}$ Onera - The French Aerospace Lab, F-92322 Châtillon, France; \\ ${ }^{\mathrm{b}}$ University of Dayton, School of Engineering, Intelligent Optics Laboratory \\ 300 College Park College Park Center, LOCI, Dayton, OH, 45469-2951 \\ ${ }^{c}$ TNO, PO Box 96864, NL-2509 JG The Hague, The Netherlands; \\ ${ }^{\mathrm{d}}$ Fraunhofer IOSB, Forschungsinstitut für Optronik, Systemtechnik und Bildauswertung, \\ Gutleuthausstr. 1, D-76257 Ettlingen; \\ ${ }^{\mathrm{e}}$ Norwegian Defence Research Establishment (FFI), Instituttveien 20, N-2007 Kjeller, Norway; \\ ${ }^{\mathrm{f}}$ U. S. Air Force Research Laboratory, Space Vehicles Directorate, 3550 Aberdeen Ave SE, \\ Kirtland AFB, NM 87117
}

\begin{abstract}
The performance of optical systems is degraded by atmospheric turbulence. Over propagation distances that exceed several kilometres, it is difficult to evaluate its impact because of terrain variability - a factor that should be taken into account. However, to optimize performance, the turbulence characteristics and its effect on optical wave propagation along the propagation path should be known. The understanding of turbulence impact is one of the main objectives of the NATO group SET 165: "Adaptive Optics (AO) for laser beam delivery, passive and active imaging and turbulence mitigation". In this paper we describe experiments performed by the NATO SET 165 research group, namely, a set of atmospheric experiments over a $7 \mathrm{~km}$ distance, and discuss some preliminary results of data processing. The experiments were conducted at the University of Dayton Intelligent Optics Laboratory (UD/IOL) in October 2011. It benefited significantly from the available optical setups and the infrastructure on the UD/IOL site.
\end{abstract}

Keywords: Turbulence, propagation, experiments, image processing

\section{INTRODUCTION}

Over propagation distances of several kilometers and above the ground, atmospheric turbulence can have a severe impact on performance of electro-optics (EO) systems. Variability of optical turbulence makes analysis and prediction of its impact difficult. In the weak turbulence regime, a formalism based on the Kolmogorov turbulence and the Rytov approximation can be applied to describe most of the optical phenomena related to the turbulence effect on propagation of optical waves in the atmosphere [1]. But for propagation near the ground over horizontal and slant propagation paths characterized by strong scintillations, the classical atmospheric turbulence models may not be sufficient to describe and correctly predict optical turbulence effects [2]. To overcome these difficulties, heuristic and statistical models were developed but their application is also limited as well as the numerical (Monte Carlo based) computer simulations that utilize a phase screen (split operator) approach [3] [4] [5].

The aim of our experiments was to acquire representative data in the strong turbulence regime and analyze them to be able to characterize and describe the effects of turbulence on imaging and laser-based system. Additionally, we wish to propose models for EO systems performance evaluation.

The experiments were conducted at the UD/IOL atmospheric propagation test-bed between 7 and 12 October 2011. The obtained experimental data include:

- Spatial and temporal intensity fluctuations in the pupil plane at different wavelengths in order to characterize the turbulence impact on pupil and focal plane intensity distributions, focal plane images are simultaneously recorded to analyze laser beam propagation;

Atmospheric Propagation IX, edited by Linda M. Wasiczko Thomas, Earl J. Spillar, Proc. of SPIE Vol. 8380 $83800 \mathrm{~J} \cdot$ ㅇ 2012 SPIE $\cdot$ CCC code: 0277-786X/12/\$18 · doi: 10.1117/12.920657 
- Images of several targets, extended and point-like, fixed and mobile, in the visible range and for different aperture sizes to evaluate atmospheric turbulence on image formation and potentials for post data -processing improvement;

- Shack-Hartmann (SH) wavefront sensor data to estimate the performance of the sensor in such conditions for AO systems.

Here we first describe the experimental setup and then present and discuss recorded data, and finally outline preliminary data post processing results.

\section{EXPERIMENTAL SET-UP}

The propagation geometry setting is shown in Figure 1. The targets and the beacons are installed on the roof of the Veterans Administration Medical Center (VAMC) building at $40 \mathrm{~m}$ high. The receivers are located $7 \mathrm{~km}$ away on the $5^{\text {th }}$ floor of the College Park center (about $15 \mathrm{~m}$ high), in the UD/IOL experimental room.

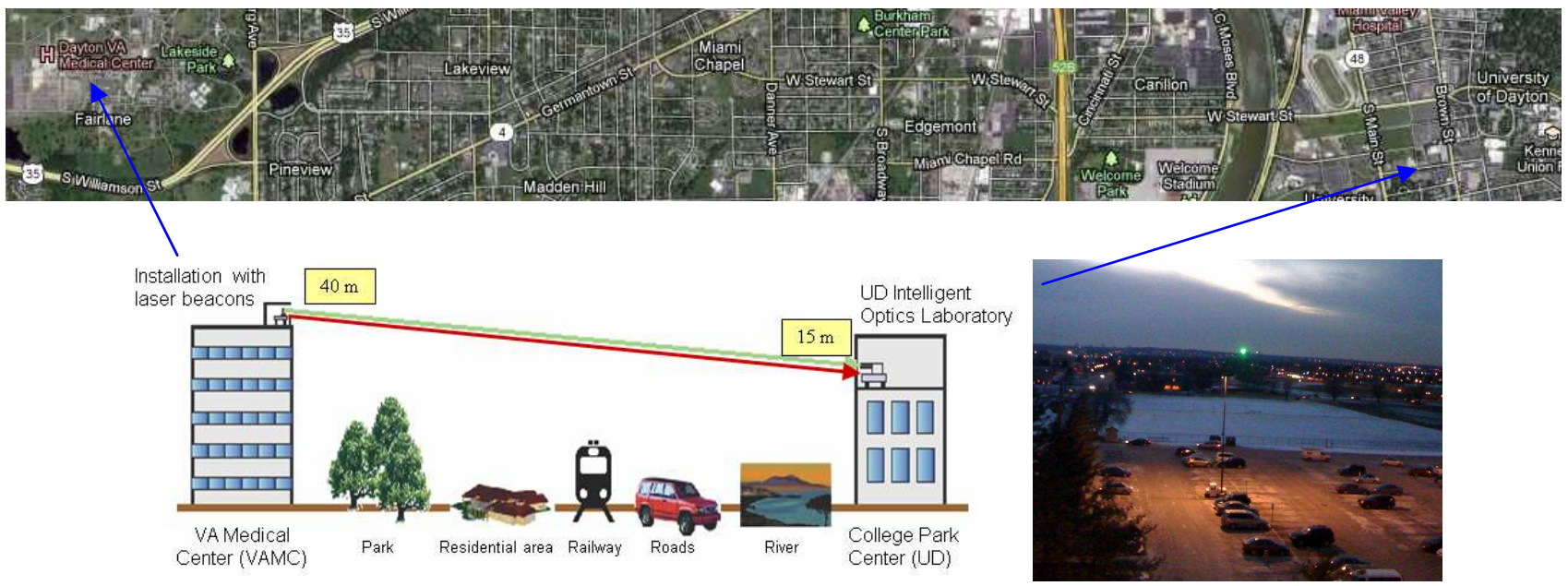

Figure 1. On top, an aerial view of the experimental site. Bottom, a graphical representation of the $7 \mathrm{~km}$ long atmospheric propagation path experimental setting (left) and snapshot of green laser beacon image taken from the receiver site at UD/IOL.

To record pupil and focal plane short-exposure intensity distributions, the COMBAT (Coherent Multi-Beam Atmospheric Transceiver) setup was used [6]. The laser beacon assembly is composed of three single-mode fiber collimators (each with a clear aperture of $26 \mathrm{~mm}$ ) and the corresponding fiber-coupled laser diodes at wavelengths of $\lambda_{1}=0.532 \mu \mathrm{m}, \lambda_{2}=1.064 \mu \mathrm{m}$ and $\lambda_{3}=1.55 \mu \mathrm{m}$. The fiber collimators are mounted together with an aiming telescope in a gimbaled system (see left side of Figure 2 below) with a smallest angular step size of $1.75 \mu \mathrm{rad}$ that is used for alignment. The beacon platform was located on an optical table inside a shed that was anchored to concrete slabs on the roof of the VAMC building.

Targets for passive imaging are a pair of airport torchlights used as white point-like sources, a checkerboard shown in Figure 2, a mannequin, and persons standing or moving on the roof of the VAMC.
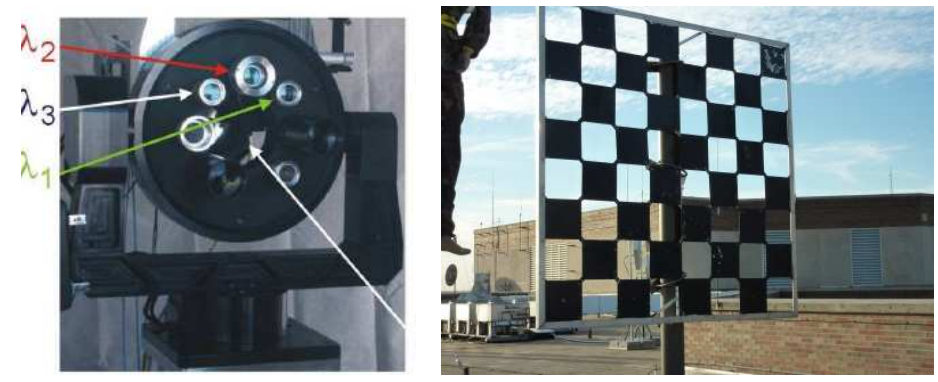

Figure 2. Left: the three laser beacons mounted on the gimbaled system; Right: the test pattern used for imaging applications. 
The COMBAT receiver system at UD/IOL is illustrated in Figure 3. After propagation to the receiver site, optical waves from the beacons are collected by a $0.35 \mathrm{~m} \mathrm{Schmidt-Cassegrain} \mathrm{telescope} \mathrm{and} \mathrm{demagnified} \mathrm{by} \mathrm{an} \mathrm{optical} \mathrm{relay} \mathrm{system.}$ The resulting collimated beam of diameter $10 \mathrm{~cm}$ is subdivided into separate subapertures, each having a usable diameter of $25 \mathrm{~mm}$, and sent to the receiver modules as shown in Figure 3. Each subaperture corresponds to an approximately 8.5 $\mathrm{cm}$ large sub-pupil of the telescope aperture. The angular dimension of the corresponding receiver subapertures are 12.1 $\mu \mathrm{rad}$. Three separate subapertures are used to record pupil-plane and focal-plane short-exposure irradiance distributions of the received beams, as shown in Figure 3. Narrow bandpass filters are used to dedicate each receiver module to a specific wavelength from a single beacon. The pupil-plane and focal intensity distributions are recorded using three Sensors Unlimited (SU640SDWHvis) 14-bit cameras. The cameras operate in a windowed mode with a $256 \times 256$ pixel window with pixel size of $25 \times 25 \mu \mathrm{m}^{2}$, providing an actual sensor area of $6.4 \times 6.4 \mathrm{~mm}^{2}$ and a $100 \%$ fill factor. A four-toone beam reducer is installed in the optical train of each subaperture to match the beam (pupil) size with the camera active window size.
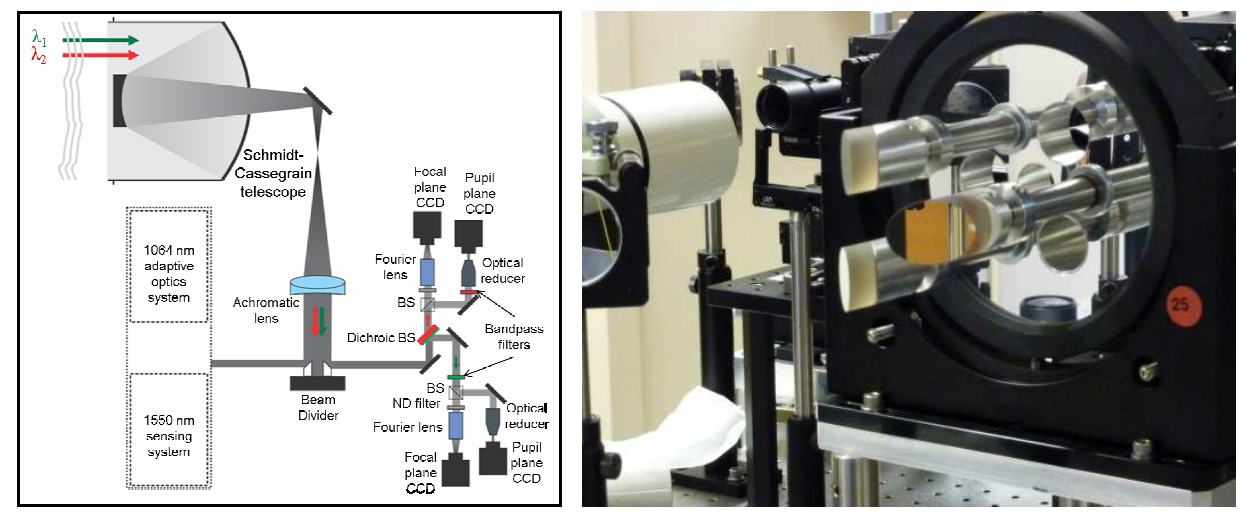

Figure 3. Left: Optical schematic of the COMBAT receiver system. The adaptive optics module was not included in the experiments. Right: the beam divider module.

The images of the targets were obtained with a Celestron 8" telescope coupled with an AVT Stingray F-080B 14-bit camera. The focal length of the telescope equals $2 \mathrm{~m}$ and the pixel size of the camera is $4.65 \times 4.65 \mu \mathrm{m}^{2}$. Its $1032 \times 778$ pixels correspond to a $4.8 \times 3.6 \mathrm{~mm}^{2}$ area. The frame rate varies from $31 \mathrm{fps}$ (frames per second) at full resolution to about 100-120 fps for smaller frames. The Celestron C8 was equipped with a variable set of off-axis diaphragms ranging from $20 \mathrm{~mm}$ to $66 \mathrm{~mm}$.

A Shack-Hartmann (SH) wavefront sensor (SHSCam from Optocraft) with a set of lenslet arrays of different sizes $(14 \times 14,144 \mu \mathrm{m}$ pitch; $11 \times 11,200 \mu \mathrm{m}$ pitch; $8 \times 8,248 \mu \mathrm{m}$ pitch; $7 \times 7,344 \mu \mathrm{m}$ pitch) was used for wavefront measurements. The SH sensor clear aperture is $2.3 \mathrm{~mm}$ and the photo- array resolution is $256 \times 256$ pixels. The SH sensor can operate with frame rate higher than $1000 \mathrm{fps}$. This sensor was located in the COMBAT sub-system operating at 1.06 $\mu \mathrm{m}$ wavelength. The sensor aperture corresponded to approximately $30 \mathrm{~mm}$ sub-aperture of the receiver telescope. During the trial, an average $\mathrm{C}_{\mathrm{n}}{ }^{2}$ along the propagation was measured with a scintillometer, as shown in figure 4 .

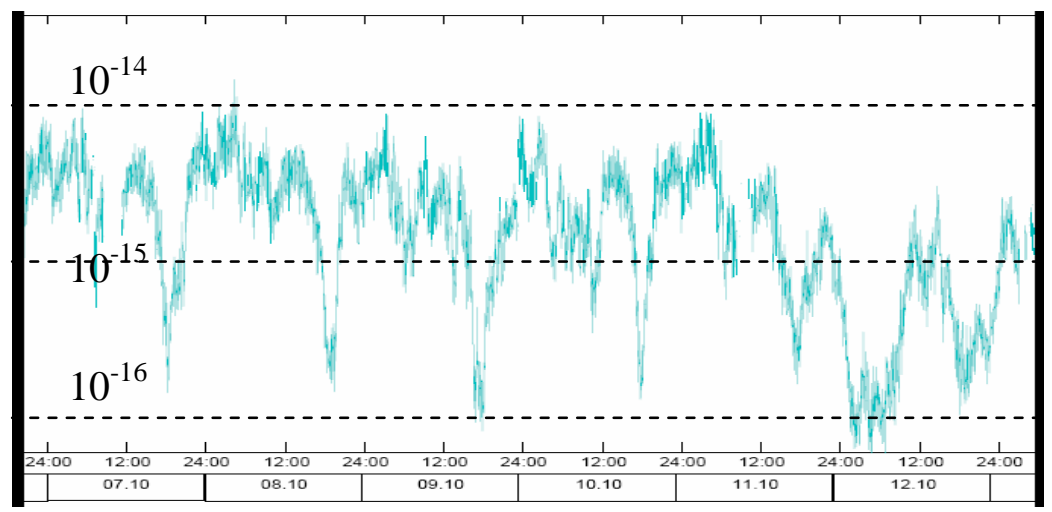

Figure 4. The example of time evolution of the refractive index structure parameter $\mathrm{C}_{\mathrm{n}}^{2}\left(\mathrm{~m}^{-2 / 3}\right)$ recorded during the trial. 


\section{MEASUREMENTS AND DISCUSSION}

\subsection{Pupil and focal plane images}

\section{Pupil plane data analysis}

Both focal and pupil plane intensity distributions were simultaneously recorded for three beacon wavelengths with frame rate varying from 200 to 400 frames/second. Examples of the pupil plane intensity distributions are shown in Fig. 5. The images show that the spatial scale of intensity scintillations depends on the wavelength, i.e., the longer the wavelength the larger the intensity speckle size. We can also observe that the dynamics of the intensity scintillation increase with the level of turbulence and decrease with the wavelength. These observations are consistent with the theoretical findings from standard turbulence theory.

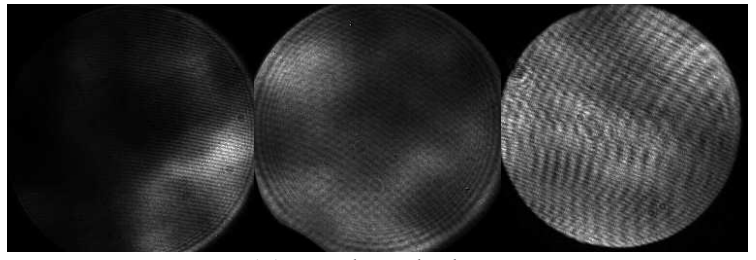

(a) weak turbulence

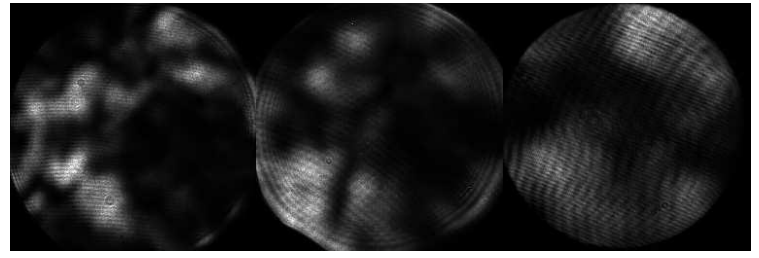

(b) strong turbulence

Figure 5. Examples of short-exposure intensity scintillation patterns recorded during experiments. From left to right the wavelength beacon is $0.532 \mu \mathrm{m}, 1.064 \mu \mathrm{m}$ and $1.55 \mu \mathrm{m}$. Turbulence level increases from the left to the right of the figures.

The temporal fluctuations of the intensity both at the centre of the pupil and summed on the whole pupil were analyzed. They depend on the wavelength and turbulence strength. The data in Fig. 6 show temporal dynamics of the scintillation index (SI), defined as $S I=\left\langle I_{P}^{2}\right\rangle_{P} /\left\langle I_{P}\right\rangle_{P}^{2}-1$, where \langle\rangle$_{\mathrm{P}}$ is the average over the pupil (summation over all the points $\mathrm{P}$ of the pupil) and $\mathrm{I}_{\mathrm{P}}$ the intensity at the point $\mathrm{P}$.
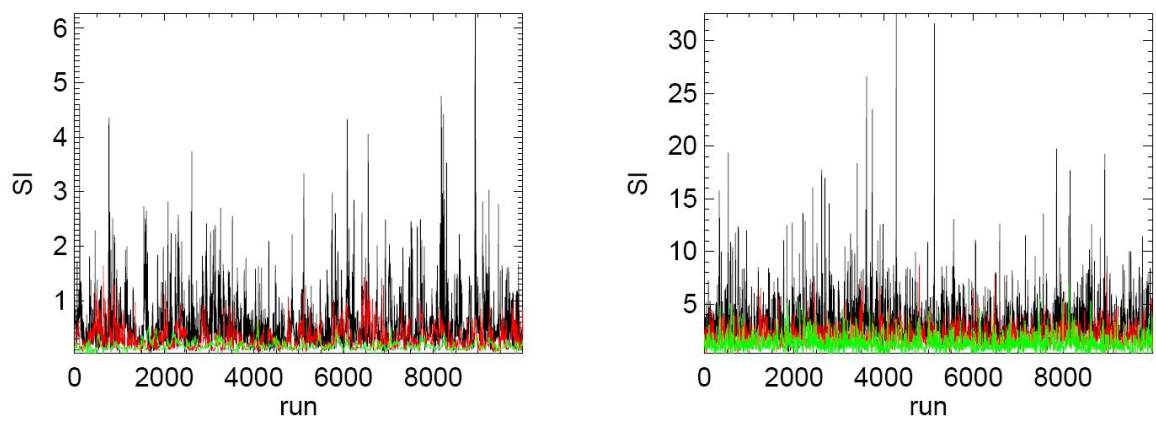

Figure 6 : Temporal dynamics of scintillation index, $\lambda=0.5$ (black), 1.06 (red), 1.55 (green). Turbulence strength increases from left to right.
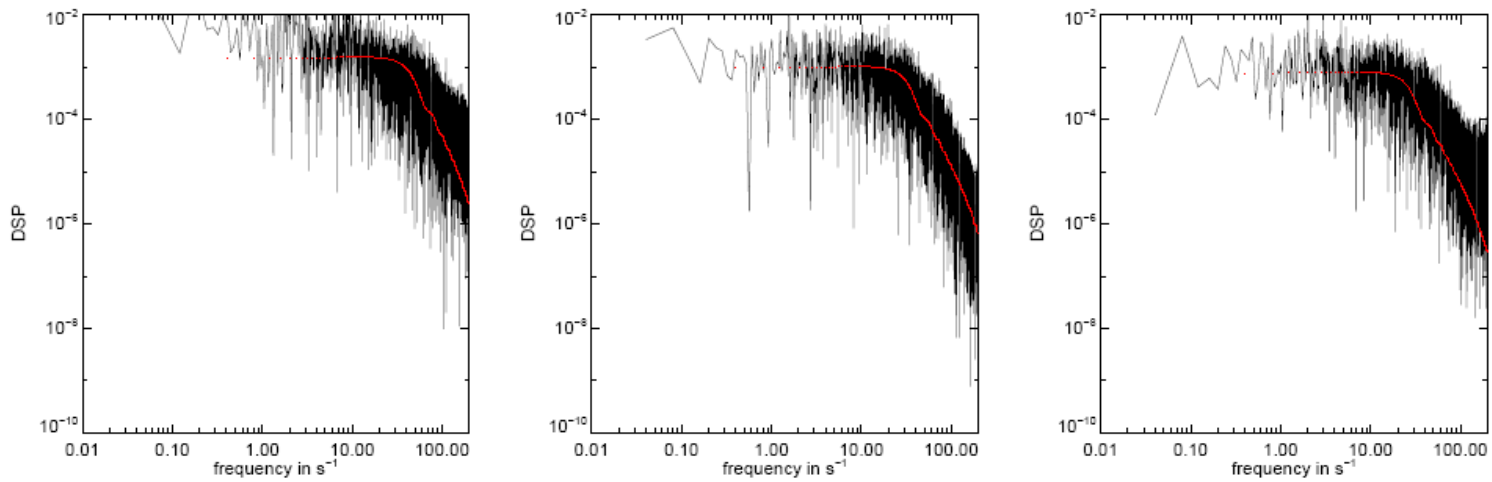

Figure 7 : Temporal spectrum of intensity fluctuations at the centre of the pupil. Wavelength increases from left to right $(0.5$, 1.06 and $1.55 \mu \mathrm{m})$. Black curves are obtained with the recorded data and red curves by an analytical approach. 
Examples of the intensity fluctuations temporal spectra at the center of the pupil are shown in Figure 7 for the three wavelengths. The spectrum obtained with the data is compared with a theoretical spectrum derived from the formalism proposed by Robert et al [7]. This formalism is valid in the Rytov regime, which is not always our application case. Most of the time, the scintillation index exceeded 1. - a commonly used limit given for the validity of Rytov's approximation [2]. However, even for strong scintillations, it seems that the Rytov approximation describes well the recorded data. We assumed an average wind speed along the line of sight of $2 \mathrm{~ms}^{-1}$ which is a little less than those expected from focal plane analysis $\left(\sim 3.5 \mathrm{~ms}^{-1}\right)$.

\section{Focal plane data}

Examples of instantaneous intensity distributions recorded at the COMBAT system focal planes are shown in Figure 8. The focal spot at the longer wavelength is larger and not regular even when the level of turbulence is lower because of residual aberrations like coma or astigmatism. When turbulence increases, spots are larger and split into several speckles.

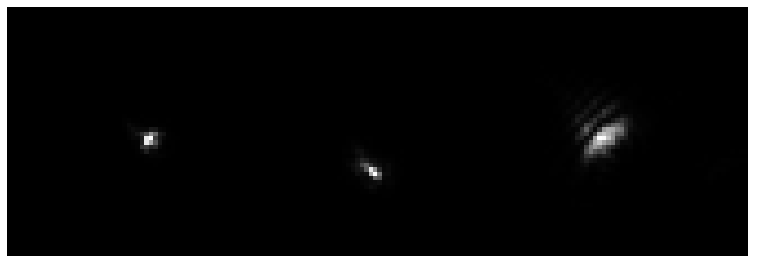

(a) weak turbulence

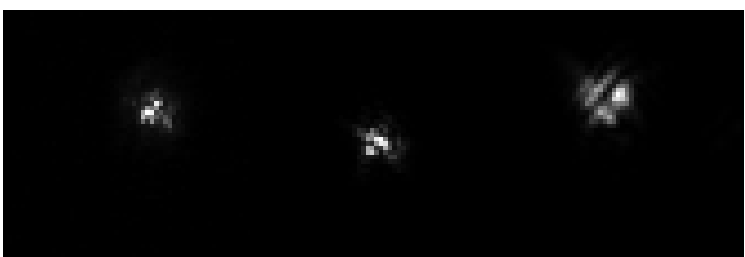

(b) strong turbulence

Figure 8. Examples of the short-exposure focal spots recorded during experiments. From left to right the wavelength beacon is $0.532 \mu \mathrm{m}, 1.064 \mu \mathrm{m}$ and $1.55 \mu \mathrm{m}$ and the turbulence level increases between left and right.

In the focal plane, short and long exposure images of the point-like sources are analyzed. The center of gravity of each short exposure image is computed and the fluctuations of the angle of arrival are derived. The corresponding temporal power spectrum is presented in Figure 9. The spectrum decay varies as $v^{-2 / 3}$ for low frequencies and $v^{-11 / 3}$ for high frequencies, which matches well with the Kolmogorov turbulence model predictions and Taylor's frozen turbulence hypothesis [8].
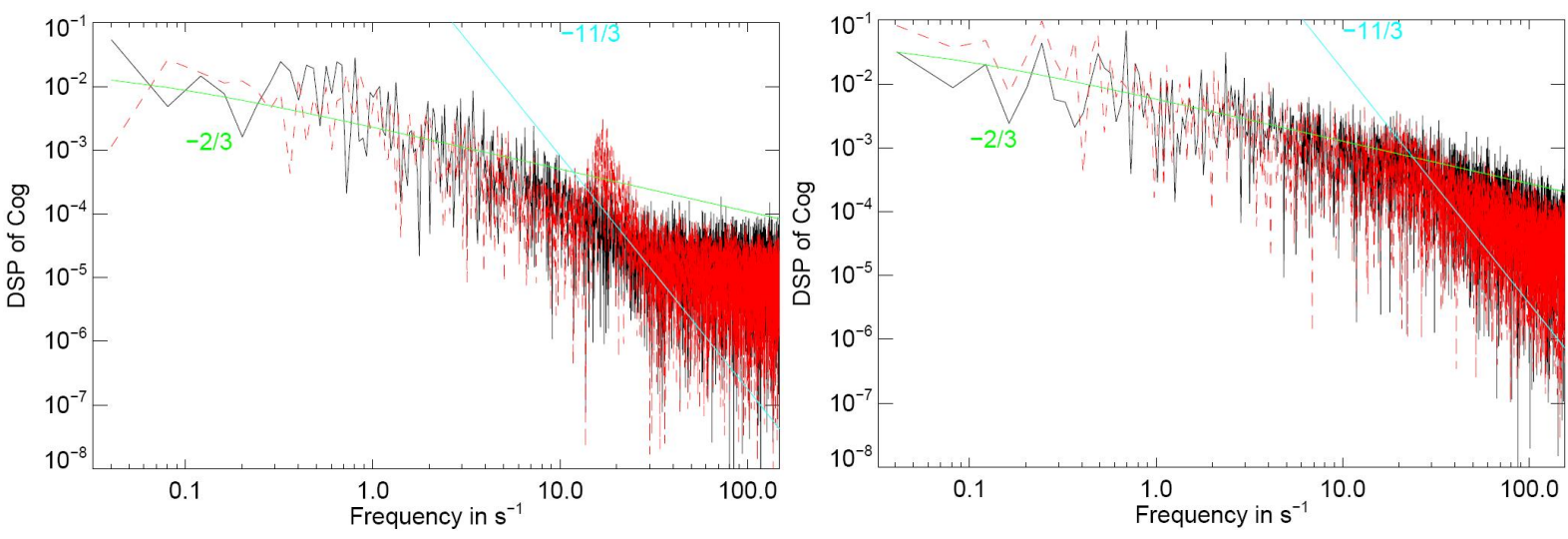

Figure 9. Temporal spectrum of the angle of arrival: Left in the case of low turbulence level $\left(\mathrm{C}_{\mathrm{n}}^{2}<10^{-15} \mathrm{~m}^{-2 / 3}\right)$, Right for a high turbulence level $\left(\mathrm{C}_{\mathrm{n}}^{2}>610^{-15} \mathrm{~m}^{-2 / 3}\right)$. The black curve represents the horizontal axis $(\mathrm{X})$ and the red curve the vertical axis $(\mathrm{Y}) ; \lambda=1.05 \mu \mathrm{m}$, frame rate $400 \mathrm{fps}$.

An estimation of the average transverse wind speed $\left(V_{w s}\right)$ can be obtained from the data presented in Figure 9. The transverse wind speed equals $v_{c} D / 0.3$, where $v_{c}$ is the intermediate cut-off frequency and D the pupil size [8]. For the two cases presented in Figure 9, estimated wind speeds are $2.5 \mathrm{~ms}^{-1}$ (left curve) and $5 \mathrm{~ms}^{-1}$ (right curve). When the turbulence effect is weaker (left curves), a frequency peak appears around $20 \mathrm{~Hz}$ for the $\mathrm{Y}$ axis. It may be due to vibrations of the reception bench, or the building. This effect becomes negligible with the increase of turbulence strength (right curves). 
In the presence of turbulence, the full width at half maximum (FWHM) of the long exposure focal plane image equals $\lambda \mathrm{f} / \mathrm{r}_{0}$ where $\mathrm{f}$ is the focal length of the optical system and $\mathrm{r}_{0}$ the Fried diameter. $\mathrm{r}_{0}$ depends on the profile of the structure parameter, noted $\mathrm{C}_{\mathrm{n}}{ }^{2}$, along the propagation path. Some analytical models were proposed to take into account the variation of $\mathrm{C}_{\mathrm{n}}{ }^{2}$ along the line of sight. For astronomical applications, the Hufnagel-Valley model can be considered. For slant paths propagations near the ground (altitude $h>10 \mathrm{~m}$ and < few hundred meters), the structure parameter $\mathrm{C}_{\mathrm{n}}{ }^{2}$ is expected to vary as $h^{-2 / 3}$ or $h^{-4 / 3}$ for stable/unstable atmospheric conditions, respectively [9]. In general, the atmosphere is considered to be stable during nighttime $\left(h^{-2 / 3}\right.$ decay) and unstable during daytime ( $h^{-4 / 3}$ decay). Because the laser beacon is quite divergent, the expression for $\mathrm{r}_{0}$ should correspond to a spherical wave approximation and is given by

$$
r_{0}=\left(0.423\left(\frac{2 \pi}{\lambda}\right)^{2} C_{n}^{2}\left(h_{0}\right) h_{0}^{p} \int_{0}^{L}\left(\frac{z}{L}\right)^{5 / 3} h^{-p}(z) \mathrm{dz}\right)^{-3 / 5} \quad[\mathrm{~m}]
$$

where exponent $p$ equal to $2 / 3$ or $4 / 3, \mathrm{~h}$ is the height above the ground along the propagation path and $h_{0}$ is a reference height where the value of $\mathrm{C}_{\mathrm{n}}{ }^{2}$ is known. The measurement of $\mathrm{r}_{0}$ using the long exposure image PSF leads to an evaluation of $\mathrm{C}_{\mathrm{n}}^{2}\left(\mathrm{~h}_{0}\right)$ using eqn (1).

The standard deviation of the angle of arrival depends also on $r_{0}$ and the outer-scale of the turbulence $\mathrm{L}_{0}$ [10]. An evaluation of these two parameters can be done with the assumption of a specific turbulence model. The commonly used is the Von-Karman model which integrates inner and outer scales.

As we mentioned above, in the weak turbulence regime, the estimation of $r_{0}$ is biased by a vibration effect. So we just analyzed data recording in stronger turbulence cases. In these cases, the estimation of $C_{n}{ }^{2}$ from $r_{0}$ measurement fits quite well with the $\mathrm{C}_{\mathrm{n}}{ }^{2}$ measurements recorded by a scintillometer (see Table 1). Due to residual aberrations for the higher wavelength as mentioned before, the long exposure focal plane image is larger and not symmetrical. This explains the overestimation of $r_{0}$ for this $\lambda$. The dependency of the standard deviation of the angle of arrival with the wavelength is low, which is consistent with the theory.

So for tactical applications (slant path near the ground), the hypotheses concerning the $\mathrm{C}_{\mathrm{n}}{ }^{2}$ profile and its spectrum seem to be realistic.

Table 1. Estimation of $r_{0}$, standard deviation of the angle of arrival $\left(\sigma_{b x}-\sigma_{b y}\right)$ and $C_{n}{ }^{2}$ estimated for the three wavelengths (blue:0.5, green:1.06 and red:1.5 $\mu \mathrm{m}$ ).

\begin{tabular}{|l|c|c|c|c|c|l|}
\hline & Lambda $(\mu \mathrm{m})$ & $\begin{array}{c}\text { Frame } \\
\text { rate }\end{array}$ & $\begin{array}{c}\mathrm{r}_{0} \text { measured } \\
(\mathrm{cm})\end{array}$ & $\begin{array}{c}\sigma_{\mathrm{bx}}-\sigma_{\text {by }} \\
(\mu \mathrm{rd})\end{array}$ & $\mathrm{C}_{\mathrm{n}}^{2}$ estimated $\left(\mathrm{m}^{-2 / 3}\right)$ & \multicolumn{1}{|c|}{$\begin{array}{c}\mathrm{C}_{\mathrm{n}}^{2}\left(\mathrm{~m}^{-2 / 3}\right) \\
(\mathrm{scintillometer})\end{array}$} \\
\hline Case 1 & $0.5-1.06-1.5$ & $205 \mathrm{fps}$ & $1-2-2.5$ & $18-15-18$ & $10^{-14}-10^{-14}-1.710^{-14}$ & $810^{-15}$ \\
\hline Case 2 & $0.5-1.06-1.5$ & $307 \mathrm{fps}$ & $1.1-2.4-2.5$ & $16-14-17$ & $810^{-15}-810^{-15}-1.610^{-14}$ & $610^{-15}$ \\
\hline Case 3 & $0.5-1.06-1.5$ & $405 \mathrm{fps}$ & $1-2-2.5$ & $18-15-18$ & $10^{-14}-10^{-14}-1.710^{-14}$ & $810^{-15}$ \\
\hline
\end{tabular}

\subsection{Target images}

In Figure 10, simultaneously recorded images of the resolution chart and a point source are shown. From Figure 10(A) to Figure 10(B), the aperture size of the telescope (and so the $\mathrm{D} / \mathrm{r}_{0}$ ratio) is reduced by a factor of 3 . We can see the influence of the pupil size, or more precisely, of the ratio $\mathrm{D} / \mathrm{r}_{0}$ on the image quality and the point spread function. As mentioned, $\mathrm{r}_{0}$ can be determined from these spot images.
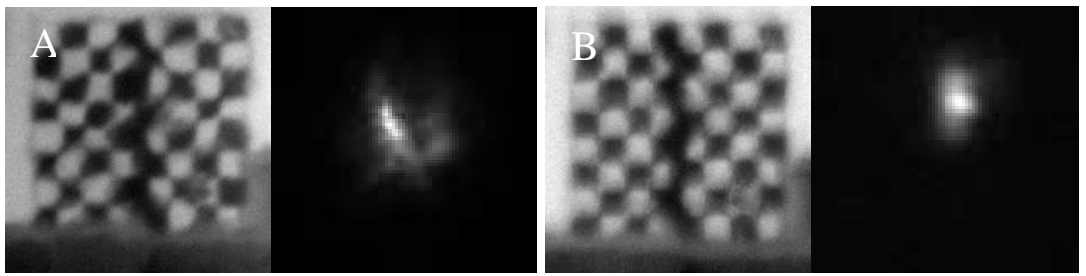

Figure 10. Examples of short-exposure images recorded during experiments with different aperture sizes, the image of the pattern and a point like source. Left: full aperture is used; Right: the aperture is $1 / 3$ of the full aperture.

\subsection{Application of image correction techniques}

One of the objectives of the study is to analyze the advantages of image correction techniques in turbulence mitigation. Two different image sequences from the recorded dataset are processed for this purpose. The method is described in 
details in [11] and consists of frame selection, registration and de-blurring. The main steps of this method are listed hereafter. A reference image is calculated by adding the $\mathrm{N}_{0}$ first images of the sequence. $\mathrm{N}_{t}$ consecutive images are choose in the sequence and the $\mathrm{N}_{\mathrm{s}}$ best frames of these $\mathrm{N}_{\mathrm{t}}$ images are selected. Local motions are evaluated on these $\mathrm{N}_{\mathrm{s}}$ images. A cost function is finally minimized to estimate the real observed target. For that, a linear model is developed to describe the image formation. It takes into account turbulence effect and optical system filtering (optics and detector). $\sigma_{\mathrm{tot}}$ and $\lambda_{\mathrm{r}}$ are parameters of this cost function.

The first sequence is captured at 12 October 2011 at $15.35 \mathrm{~h}$ and contains a building with the word "NATO" attached to it. The distance over which the image is taken, is approximately $7 \mathrm{~km}$. The refractive index structure parameter was measured at that time as $C_{n}^{2}=6.9 \times 10^{-16} \mathrm{~m}^{-2 / 3}$. The camera used is the AVT Stingray F-080B with a maximum frame size of $1032 \times 778$ pixels and a pixel depth of 14-bit monochrome. For the optics a Celestron C8 is used (see Section 2 for details). The "NATO" sequence is captured with a resolution of $1032 \times 264$ pixels, a frame rate of $69 \mathrm{~Hz}$, a shutter time of $25 \mathrm{~ms}$ and a camera gain of $16.2 \mathrm{~dB}$.

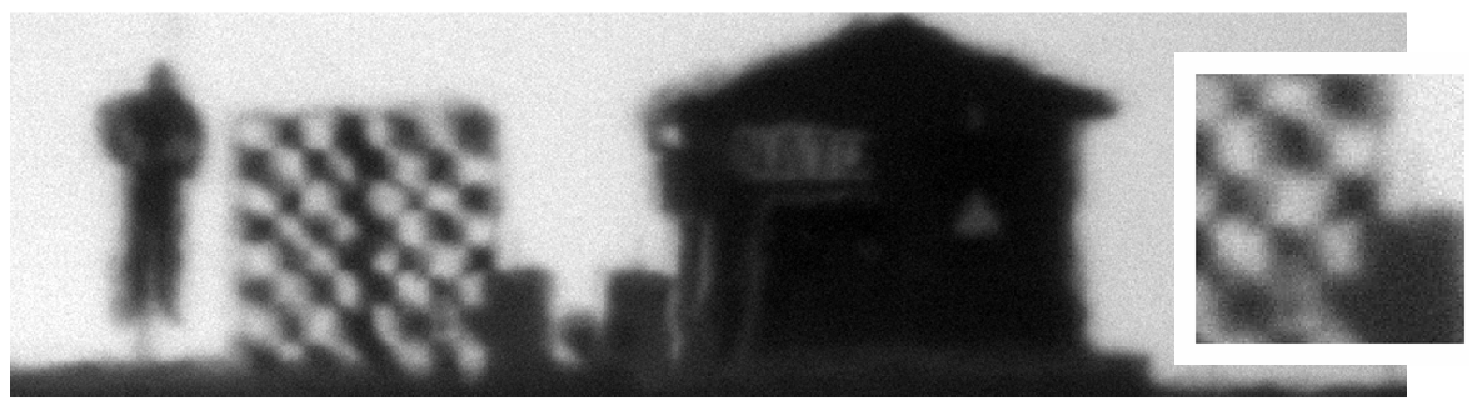

(a)

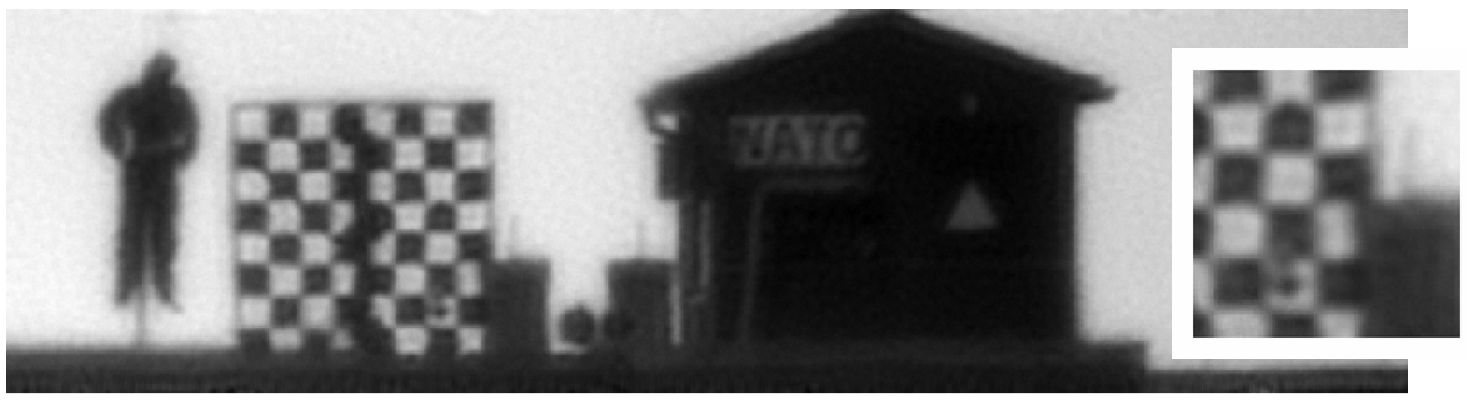

(b)

Figure 10 (a) One of the blurred frames of the "NATO" sequence taken on 12 October 2011 at $15.35 \mathrm{~h}$ with a detailed inset at the right. (b) Corresponding processed frame by one method described in [5].

The following parameter settings were used to process 200 frames: $N_{0}=20$ frames, $N_{t}=20$ frames, $N_{s}=10$ frames, resampling factor 1 , blurring $\sigma_{\text {tot }}=3, \lambda_{r}=10^{-3}$ and 10 iterations for the minimization procedure. One of the processed frames is shown together with an input frame in Figure 10 (a), although the result can be appreciated best by watching a video. Looking at the images in Figure 10 (b), it is clear that more detail is visible in the processed frame, such as on the checkerboard and the NATO sign on the building.

Another sequence from the Dayton dataset showing an antenna tower gives even more impressive performance. This sequence was captured on 12 October 2011 at $16.15 \mathrm{~h}$ with a resolution of $552 \times 776$ pixels, a frame rate of $31.6 \mathrm{~Hz}$, a shutter time of $8.3 \mathrm{~ms}$ and a camera gain of $22.7 \mathrm{~dB}$. The refractive index structure parameter was measured at that time as $C_{n}^{2}=8.2 \times 10^{-16} \mathrm{~m}^{-2 / 3}$.

The following parameter settings were used to process 200 frames: $N_{0}=20$ frames, $N_{t}=20$ frames, $N_{s}=10$ frames, resampling factor 1 , blurring $\sigma_{\mathrm{tot}}=3, \lambda_{r}=0.1$ and 10 iterations for the minimization procedure. Input frame 100 is shown together with the corresponding processed frame. Here it is very clear that processing helps to visualize more details. Results are shown in Figure 11. 


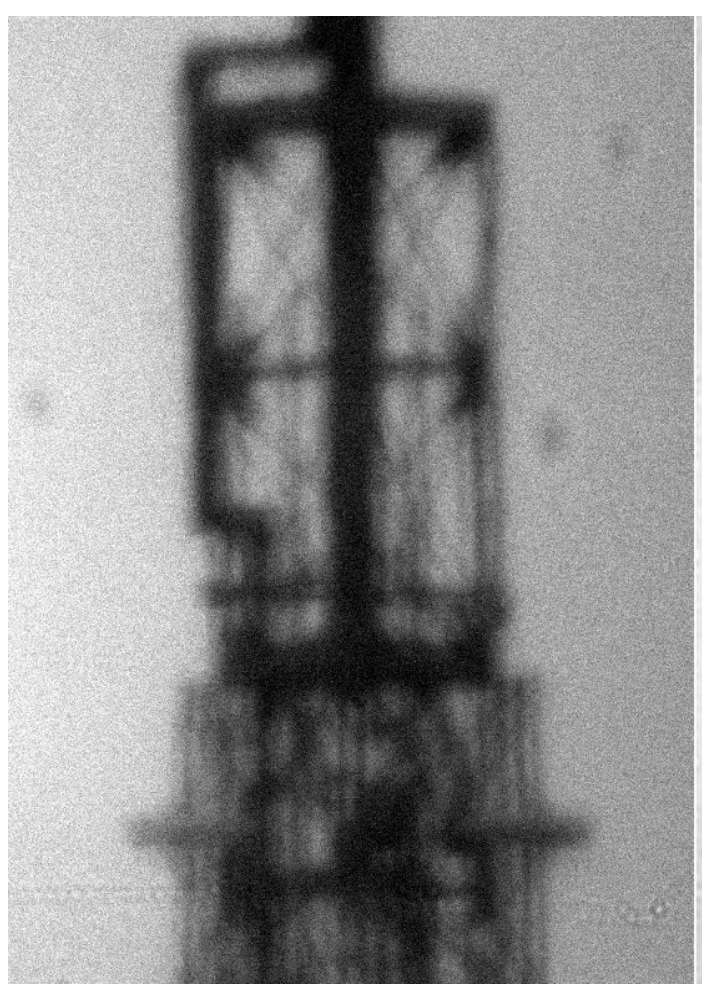

(a)

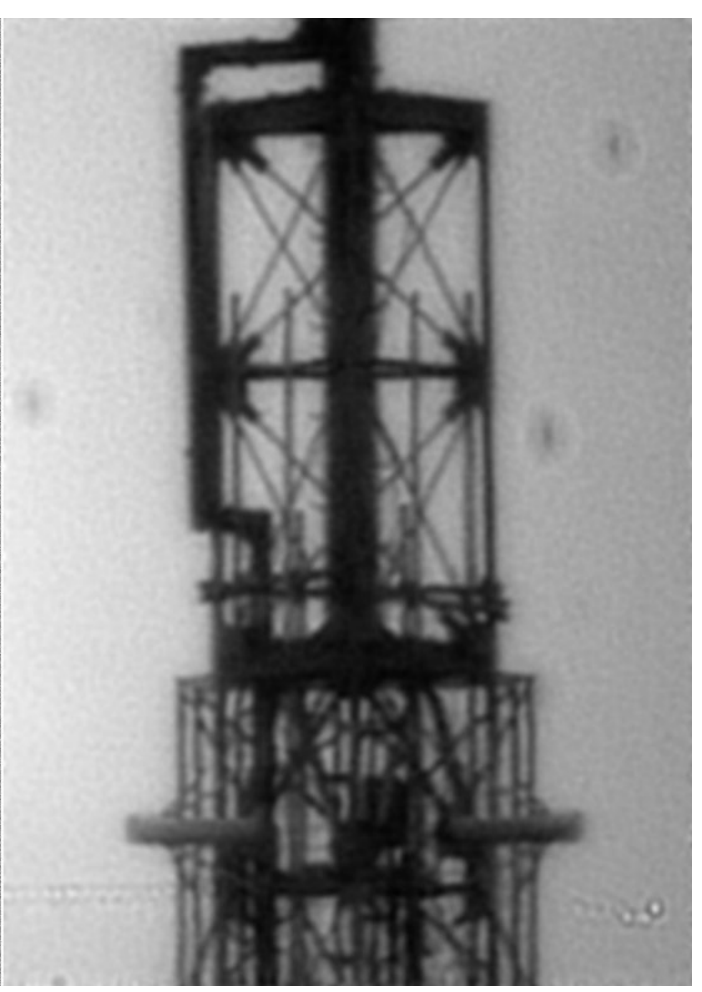

(b)

Figure 11 (a) One of the blurred frames of the "Antenna" sequence taken on 12 October 2011 at 16.15h. (b) Corresponding processed frame by one method

\subsection{Wavefront sensor data}

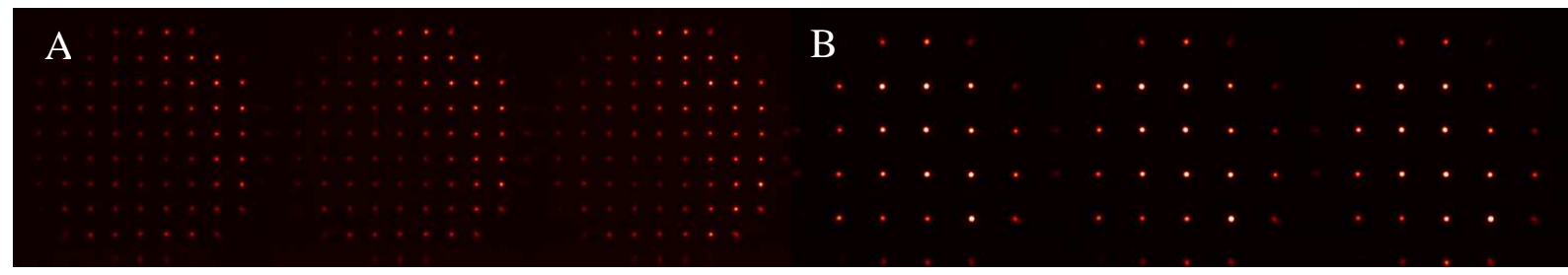

Figure 12. Examples of data recorded in the focal plane of the SH sensor during experiments with two sets of lenslet arrays, $\lambda=1.05 \mu \mathrm{m}$. The time between the frames is $1 \mathrm{~ms}$.

Figure 12 shows the sampling of the wavefront by two sets of lenslet arrays with decreasing spatial frequencies (or decreasing the number of subapertures and increasing their size). In Figure 12 (A), we see that scintillation results in low intensity levels for focal spots. A lenslet array with fewer subapertures is less sensitive to scintillation, as intensity fluctuations are averaged over larger subaperture areas, as illustrated by Figure 12 (B).

For strong scintillation, a SH sensor with high spatial resolution doesn't perform well. The accuracy of wavefront sensing can be improved by using a lenslet with a larger diameter for individual lenses. But this results in lower spatial resolution in wavefront sensing. More analysis must be done to find a trade off between scintillation minimization and correct phase measurements.

\section{CONCLUSION}

During the atmospheric turbulence characterization trial, a large amount of data was recorded. Preliminary results obtained from the processing of the trial data show a good agreement with predictions from turbulence theory, including 
the Rytov approximation. Further work consists of completing the processing of the data to confirm the turbulence models we have proposed here or to present their limitations.

We have shown results of compensation of the effects of turbulence in static image sequences. The results show that the correction method is capable of stabilizing and de-blurring such image sequences. Also, it is shown that resolution enhancement is possible in images captured during weak to mild turbulence conditions. The presented method enables an operator to see more details at a large distance and it enlarges the recognition range.

\section{ACKNOWLEDGEMENTS}

The trial was partially supported by NATO RTO SET Pannel. The authors wish to thank all the personal of the Intelligent Optics laboratory at UD, UMD and the Army Research Laboratory and Optonicus LLC including Thomas Weyrauch, Ernst Polnau, Mathieu Aubailly, Gary Carhart, Jiang Liu, Richard Espinola, Andrew Deck and Tim Goode for their helpful contribution in the trial and active participation.

\section{REFERENCES}

[1] Tatarski V. I., "Wave propagation in a Turbulent Medium," McGraw-Hill Book Company, New York, (1961).

[2] Brown, JR W. P.., "Validity of the Rytov Approximation," J. Opt. Soc. Am. 57, 1539-1542 (1967).

[3] Fante, R. L., "Electromagnetic beam propagation in turbulent media," Proceedings of the IEEE, vol.63, no.12, pp. 1669- 1692, (1975).

[4] Andrews L. C., and Philipps R. L., "Laser Beam Propagation through Random Media," $2^{\text {nd }}$ ed. SPIE Optical Engineering Press, Bellingham, (2005).

[5] Martin J., and Flatté S., "Intensity images and statistics from numerical simulation of wave propagation in 3-D random media," Appl. Opt. 27, 2111-2126.

[6] Vorontsov M., Rao Gudimetla V. S., Carhart G., Weyrauch T., Lachinova S., Polnau E., Reierson J., Beresnev L., Liu J., and Riker J. F., "Comparison of turbulence-induced scintillations for multi-wavelength laser beacons over tactical $(7 \mathrm{~km})$ and long $(149 \mathrm{~km})$ atmospheric propagation paths," AMOS, Advanced Maui Optical and Space Surveillance Technologies Conference, 12-16 Sep 2011, Maui, HI (2011)

[7] Robert C., Conan J.-M., Michau V., Renard J., Robert C., and Dalaudier F., "Retrieving parameters of the anisotropic refractive index fluctuations spectrum in the stratosphere from balloon-borne observations of stellar scintillation," J. Opt. Soc. Am. A 25, 379-393 (2008).

[8] Conan J.-M., Rousset G., and Madec P.-Y., "Wave-front temporal spectra in high-resolution imaging through turbulence," J. Opt. Soc. Am. A 12, 1559-1570 (1995).

[9] Wyngaard J., Izumi Y., and Collins, JR. S., "Behavior of the Refractive-Index-Structure Parameter near the Ground," J. Opt. Soc. Am. 61, 1646-1650 (1971).

[10] Borgnino J., "Estimation of the spatial coherence outer scale relevant to long baseline interferometry and imaging in optical astronomy," Appl. Opt. 29, 1863-1865 (1990).

[11] Van Eekeren, A. W. M., Kruithof, M. C., Schutte, K., Dijk, J., van Iersel, M., and Schwering, P. B. W., "Patchbased local turbulence compensation in anisoplanatic conditions," Proc. SPIE Infrared Imaging Systems: design, analysis, modeling and testing XXIII, vol.8355 (2012) 